\section{CONSEJERÍA FARMACÉUTICA Y ADHERENCIA AL TRATAMIENTO ANTIHIPERTENSIVO: ESTUDIO PRELIMINAR}

\author{
PHARMACEUTICAL COUNSELING AND \\ ADHERENCE TO ANTIHYPERTENSIVE \\ TREATMENT: PRELIMINARY STUDY
}

\author{
Maribel Limaylla-La-Torre, $1, a, b$
}

Sr Editor. Las enfermedades crónicas no transmisibles, constituyen un grupo heterogéneo de padecimientos como diabetes, hipertensión arterial (HTA), entre otros, constituyéndose en un problema de salud pública ${ }^{(1)}$. Se reconoce que la HTA es una enfermedad de alta frecuencia en la población adulto mayor atendida en el Centro de Atención Primaria (CAP) III de Huaycán EsSalud en Lima, Perú.

Con el objetivo de evaluar el nivel de adherencia al tratamiento farmacológico, el nivel inicial de conocimiento sobre la hipertensión arterial y el nivel de satisfacción en relación a la consejería farmacéutica (CF) brindada, se realizó un estudio en una muestra de 22 adultos mayores con HTA, atendidos por el Programa del Adulto Mayor (PAM) y/o por el médico internista del CAP.

El estudio comprendió dos fases: a) la oferta del servicio de CF, que se realizó a través de la captación de pacientes en ventanilla de farmacia y la captación de los atendidos en el PAM, y b) el desarrollo de las sesiones de consejería farmacéutica ${ }^{(2)}$, en la cual se brindaron charlas de educación sanitaria a través de visitas domiciliarias, con afiches impresos para facilitar la visualización y mejorar la comprensión; además, se brindaron pautas sobre tratamiento no farmacológico.

Se evaluó la adherencia al tratamiento y el conocimiento sobre HTA al inicio y al final de la CF, para lo cual se utilizó el test de Morisky-Green y Batalla; para evaluar calidad de la CF en pacientes con HTA se utilizó un cuestionario con 17 preguntas tipo Likert con cinco dimensiones (fiabilidad, capacidad de respuesta, seguridad, empatía y tangibilidad), cuya evaluación de validez se realizó por juicio de expertos con una concordancia significativa en la prueba binomial

\footnotetext{
Universidad Nacional Mayor de San Marcos. Lima, Perú

Químico farmacéutica, ${ }^{\mathrm{b}}$ magister en Atención Farmacéutica.

Recibido: 25/01/2017 Aprobado: 08/02/2017 En línea: 28/06/2017
}

Citar como: Limaylla-La-Torre M. Consejería farmacéutica y adherencia al tratamiento antihipertensivo: estudio preliminar [carta]. Rev Peru Med Exp Salud Publica. 2017;34(2):343-4. doi: 10.17843/rpmesp.2017.342.2647 $(p<0,002)$, y una evaluación de la confiabilidad con un resultado del coeficiente $\alpha$ de Cronbach de 0,752 ; por lo que se concluyó que el instrumento elaborado fue válido y el grado de concordancia entre los jueces fue significativo, además de tener una alta confiabilidad interna y coherencia interna entre ítems.

Luego de la CF se obtuvo una mejoría significativa en la adherencia, según las diferencias de los resultados del test de Morisky-Green al inicio y al final $(p<0,05)$. Asimismo, se alcanzó mejora significativa en el nivel de conocimiento del paciente sobre la HTA, mediante la evaluación de los resultados de la prueba de Batalla al inicio y final $(p<0,05)$ (Tabla 1$)$; es decir, hubo un incremento de 36,4 puntos por ambas pruebas luego de la CF. La percepción de calidad de la CF mostro un alto nivel de satisfacción en los pacientes con HTA.

Según Gutiérrez-Angulo ML et al. ${ }^{(3)}$ existe una gran variabilidad en el cumplimiento farmacológico en diversos estudios, este valor oscila entre 7,1 y $66,0 \%$, y depende de las patologías, de las variables estudiadas y de la distribución por género de las muestras. Asimismo, Torres A et al. ${ }^{(4)}$, en un estudio similar, refieren un cambio significativo en la adherencia desde el inicio (30\%) y luego de la intervención $(60,8 \%)(p=0,01)$; con un incremento de 31 puntos, similar a nuestros hallazgos. Sin embargo, se debe reconocer que nuestro tamaño de muestra limitado no permite realizar comparaciones adecuadas con otros estudios, no obstante, estos hallazgos pueden considerarse como datos preliminares para futuras investigaciones.

Estudios mencionan que la consejería farmacéutica mejora la adherencia terapéutica a corto plazo y en pacientes hospitalizados, destaca su potencial para reducir la readmisión y, por lo tanto, los costos de la atención de salud con mejores resultados para los pacientes ${ }^{(5)}$.

Tabla 1. Efecto de la consejería farmacéutica en la adherencia al tratamiento antihipertensivo y en el nivel de conocimiento sobre hipertensión arterial en adultos mayores atendidos en el CAP III de Huaycán $(n=22)$

\begin{tabular}{|c|c|c|c|}
\hline \multicolumn{3}{|c|}{${\text { Adherencia al tratamiento antihipertensivo }{ }^{\dagger}}^{-}$} & \multirow{2}{*}{ Valor $p$ * } \\
\hline Antes de la CF & Después de la CF & $\mathrm{n}(\%)$ & \\
\hline Buena & Buena & $1(4,5)$ & 0,008 \\
\hline Mala & Buena & $8(36,4)$ & \\
\hline Mala & Mala & $13(59,1)$ & \\
\hline \multicolumn{4}{|c|}{ Conocimiento sobre la hipertensión arterial ₹ } \\
\hline Bueno & Bueno & $11(50,0)$ & 0,008 \\
\hline Regular & Bueno & $8(36,4)$ & \\
\hline Regular & Regular & $3(13,6)$ & \\
\hline
\end{tabular}


Se concluye que la CF puede ser una herramienta útil en la adherencia terapéutica en pacientes con HTA y en la mejora del nivel de conocimiento de su enfermedad; esta se podría utilizar de forma más activa con otras patologías, por lo que se sugiere la realización de más estudios al respecto.

\section{REFERENCIAS BIBLIOGRÁFICAS}

1. Organización Mundial de la Salud. Informe sobre la situación mundial de las enfermedades no transmisibles 2010 [Internet]. Ginebra: OMS; 2011 [citado el 5 enero del 2017].

2. Reglamento de Establecimiento Farmacéuticos. Decreto Supremo No 014-2011-SA. Normas Legales El Peruano (27 de julio de 2011).

3. Gutiérrez-Angulo ML, Lopetegi-Uranga P, Sánchez-Martin I, Garaigordobil-Landazabal M. Cumplimiento terapéutico en pacientes con hipertensión arterial y diabetes mellitus 2 . Rev Calid Asist. 2012;27(2):72-7. doi:10.1016/j.cali.2011.09.008.

4. Torres A, Fite B, Gasco P, Barau M, Guayta-Escolies R, EstradaCampmany M, et al. Efectividad de un programa de atención farmacéutica en la mejora del control de la presión arterial en pacientes hipertensos mal controlados. Estudio PressFarm. Hipertens riesgo vasc. 2010;27(1):13-22. doi:10.1016/j. hipert.2009.05.007

5. Al-Rashed SA, Wright DJ, Roebuck N, Sunter W, Chrystyn $\mathrm{H}$. The value of inpatient pharmaceutical counselling to elderly patients prior to discharge. Br J Clin Pharmacol. 2002;54(6):657-64. doi:10.1046/j.1365-2125.2002.01707.x.

Correspondencia: Maribel Lilia Limaylla La Torre

Dirección: Jr. Los Amautas $N^{\circ} 383$ Urbanización Zárate San Juan de Lurigancho. Lima, Perú

Teléfono: (+511) 969961553

Correo electrónico:singelb777@yahoo.com

\section{LEISHMANIOSIS CUTÁNEA VERRUCOSA: INUSUAL PRESENTACIÓN}

\section{VERRUCOUS CUTANEOUS LEISHMANIASIS: UNUSUAL PRESENTATION}

\section{Milton José Max Rodríguez-Zúñiga ${ }^{1,2}$, Jorge Idrogo-Bustamante ${ }^{1,2}$, Eberth Quijano-Gomero ${ }^{1}$}

Señor editor. La leishmaniosis es una zoonosis parasitaria producida por distintas especies del género Leishmania (L.), y transmitida en nuestro país por la picadura del díptero hematófago hembra del género

\footnotetext{
Servicio de Dermatología del Hospital Nacional Daniel Alcides Carrión, Callao, Perú.

2 Universidad Nacional Mayor de San Marcos, Lima, Perú.

Recibido: 02/02/2017 Aprobado: 08/02/2017 En línea: 30/05/2017
}

Citar como: Rodríguez-Zúñiga MJM, Idrogo-Bustamante J, Quijano-Gomero E. Leishmaniosis cutánea verrucosa: inusual presentación [carta]. Rev Peru Med Exp Salud Publica. 2017;34(2):344-5. doi: 10.17843/rpmesp.2017.342.2663
Lutzomia (Lu.) (1). Se reportan 1 a 2 millones de casos nuevos en el mundo; en el Perú, para el 2014, se reportaron 6231 casos autóctonos, $6,3 \%$ de casos menos con respecto al 2013 (6647 casos), siendo la tasa de incidencia anual de 20,4 casos por 100000 habitantes. La leishmaniosis se ha asociado con actividades económicas de riesgo tales como taladores de árboles, minería, etc. ${ }^{(2)}$.

Se clasifican clínicamente en cutánea (95\%) y mucocutánea $(5 \%)$, y según el criterio epidemiológico (procedencia) en andina y selvática. La lesión se caracteriza por aparecer en zonas de piel expuestas tales como cara y extremidades, que inician como un nódulo pequeño no doloroso, de evolución crónica y que, posteriormente, se vuelve una úlcera en "sacabocado", de bordes indurados y de fondo granulomatoso ( $96 \%$ de los casos). Por otro lado, las formas verrugosas están reportadas solo en el $0,5 \%$ de $\operatorname{casos}^{(3)}$.

En el Servicio de Dermatología del Hospital Nacional Daniel Alcides Carrión del Callao, Perú, se presentó un paciente varón de 24 años de edad, natural y procedente de Lima, con una pústula en la región glabelar, que fue progresando hasta transformarse a múltiples nódulos indoloros. Luego de dos semanas, tras falta de respuesta terapéutica a antibióticos tópicos y orales, se evidenció un tumor verrugoso de bordes eritematosos, nodulares e indoloro. (Figura 1A). En la biopsia cutánea se reportó una dermatitis granulomatosa con presencia de amastigotes en histiocitos (Figura 1B), y en el examen de reacción en cadena de la polimerasa, se informó infección por L. (Viannia) guyanensis. Los datos epidemiológicos confirmaron que el paciente viajó a la reserva natural de Yanachaga-Chemillén en Oxapampa, Pasco, tres meses antes del inicio de los síntomas. Recibió tratamiento con dosis de $20 \mathrm{mg} / \mathrm{kg}$ de peso de estibogluconato de sodio endovenoso por 20 días, que lo llevó a la resolución de la lesión (Figura 1C).

Se realiza la presente carta para reportar la inusual presentación clínica de leishmaniosis, que inicia el cuadro como un forúnculo y luego una placa verrugocostrosa, lo que condicionó al retraso diagnóstico y terapéutico. La gran variedad clínica de la leishmaniosis depende de factores como el parásito, el huésped y la respuesta inmune. Las formas atípicas e inusuales son esporotricoide, psoriasiforme, verrugosa, lupoide, erisipeloide, etc. y se observan en el 2 a $5 \%$ del total de pacientes afectados. De forma similar, se reportó un caso de leishmania verrugosa en la rodilla de un paciente, con presencia de múltiples placas eritemato costrosas infiltradas no dolorosas, de difícil diagnóstico debido a la forma atípica de presentación ${ }^{(4)}$. En estos casos se deben considerar otros diagnósticos diferenciales, tales como tuberculosis verrugosa, cromomicosis, 